\title{
KIR gene content imputation from single-nucleotide polymorphisms in the Finnish population
}

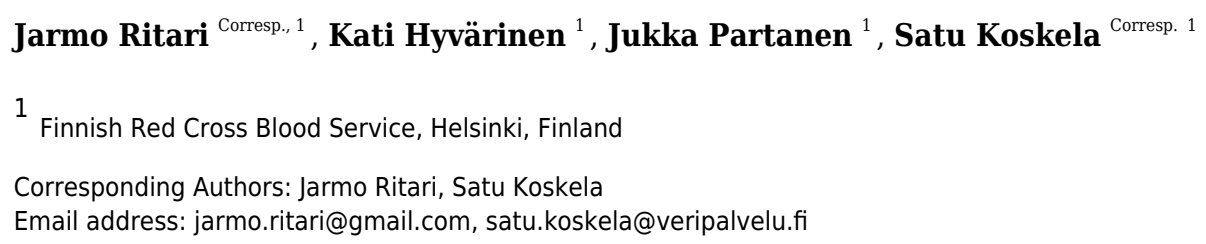

The killer cell immunoglobulin-like receptor (KIR) gene cluster on chromosome 19 encodes cell surface glycoproteins that bind class I human leukocyte antigen (HLA) molecules as well as some other ligands. Through regulation of natural killer (NK) cell activity KIRs participate in tumour surveillance and clearing viral infections. KIR gene gene copy number variation associates with the outcome of transplantations and susceptibility to immune-mediated diseases. Inferring KIR gene content from genetic variant data is therefore desirable for immunogenetic analysis, particularly in the context of growing biobank genome data collections that rely on genotyping by microarray. Here we describe a stand-alone and freely available gene content imputation for $12 \mathrm{KIR}$ genes. The models were trained using 807 Finnish biobank samples genotyped for 5900 KIR-region SNPs and analysed for KIR gene content with targeted sequencing. Cross-validation results demonstrate a high mean overall accuracy of $98.5 \%$ (95\% Cl: 97.0-99.2\%) which compares favourably with previous methods including short-read sequencing based approaches. 
1 KIR gene content imputation from single-nucleotide polymorphisms in

2 the Finnish population

3

4 Jarmo Ritari ${ }^{1}$, Kati Hyvärinen ${ }^{1}$, Jukka Partanen ${ }^{1}$, Satu Koskela ${ }^{1}$

5

$6{ }^{1}$ Finnish Red Cross Blood Service, Helsinki, Finland

7 Corresponding Author:

8 Jarmo Ritari ${ }^{1}$

9 Haartmaninkatu 8, 00290 Helsinki , Finland

10 Email address: jarmo.ritari@veripalvelu.fi

11

12 
13

14

15

16

17

\section{Abstract}

The killer cell immunoglobulin-like receptor (KIR) gene cluster on chromosome 19 encodes cell surface glycoproteins that bind class I human leukocyte antigen (HLA) molecules as well as some other ligands. Through regulation of natural killer (NK) cell activity KIRs participate in tumour surveillance and clearing viral infections. KIR gene copy number variation associates with the outcome of transplantations and susceptibility to immune-mediated diseases. Inferring KIR gene content from genetic variant data is therefore desirable for immunogenetic analysis, particularly in the context of growing biobank genome data collections that rely on genotyping by microarray. Here we describe a stand-alone and freely available gene content imputation for 12 KIR genes. The models were trained using 807 Finnish biobank samples genotyped for 5900 KIR region SNPs and analysed for KIR gene content with targeted sequencing. Cross-validation results demonstrate a high mean overall accuracy of $98.5 \%(95 \% \mathrm{Cl}$ : $97.0-$ 99.2\%) which compares favourably with previous methods including short-read sequencing-based approaches.

\section{Introduction}

Killer cell immunoglobulin-like receptors (KIRs) regulate the activity of natural killer (NK) cells and a subset of T cells via inhibitory and activating signals. Through their KIR molecules NK cells detect phenotypic change in a target cell. KIRs recognise human leukocyte antigen (HLA) class I molecules as cognate ligands, limiting to particular HLA allotypes within the serological HLA-C1 and C2 allele groups (Wroblewski, Parham \& Guethlein, 2019) HLA-Bw4 motif, HLA-A3/11, HLA-G and HLA-F (Garcia-Beltran et al., 2016). The functional difference between inhibitory and activating KIRs is determined by the presence or absence of a cytoplasmic immunoreceptor tyrosine-based inhibitory (ITIM) protein motif, respectively. In the absence of constitutive signaling conveyed by an inhibitory KIR binding to its class I ligand, NK cell cytotoxic activity and cytokine production are triggered (Lanier, 2008).

According to the missing-self hypothesis, NK cells recognise tumour or virally infected cells that attempt to evade T cell mediated immunity by downregulating their cell surface HLA-molecules that present intracellular antigens to T cells. Activating KIRs, in contrast, are thought to recognise surface molecules indicative of aberrant host cell activity such as an exceptionally high surface density of HLA class I 
molecules even though in some cases the ligand remains unknown (Ivarsson, Michaëlsson \& Fauriat, 2014). Activating KIRs have lower affinity to their ligands than inhibitory KIRs (Stewart et al., 2005) most likely owing to NK cell education to maintain self-tolerance. However, upon receiving a sufficient positive stimulus, they are able induce NK cell activation and target cell lysis. A vast majority of genetic associations of KIRs with cancer, autoimmunity and infectious diseases are attributed to variation in activating KIRs (Parham \& Guethlein, 2018).

The KIR gene cluster on the human chromosome 19q13.4 encodes fifteen relatively homologous KIR genes and two pseudogenes, constituting two main haplotypes: A and B

(https://www.ebi.ac.uk/ipd/kir/sequenced haplotypes.html). The group A haplotype consists of KIR3DL3, KIR2DL3, KIR2DL1, KIR2DL4, KIR3DL1, KIR2DS4 and KIR3DL2 genes of which all except KIR2DS4 are inhibitory. The group B haplotype, on the other hand, is more diverse being characterised by the presence of at least one of KIR2DS2, KIR2DL2, KIR2DL5, KIR2DS5, KIR3DS1, KIR2DS3 or KIR2DS1 genes (Bashirova et al., 2006). Thus, the group B haplotype harbours several genes, whereas the only activating receptor, KIR2DS4, of the group $\mathrm{A}$ is in a significant proportion of Caucasians a non-functional truncated variant (Maxwell et al., 2002; Bontadini et al., 2006), rendering about $40 \%$ of group A homozygotes solely inhibitory. Approximately $55 \%$ of haplotypes are mixtures between group A and B (Middleton \& Gonzelez, 2010), making the haplotype strucure highly variable in the population. Allelic diversity within KIRs is equally high with at least a few hundred known polymorphisms (https://www.ebi.ac.uk/ipd/kir/stats.html), which can affect class I ligand affinity (Carr, Pando \& Parham, 2005; Frazier et al., 2013).

Discovery and interpretation of KIR gene and haplotype associations in large biobank genome data collections can be facilitated by imputation of KIR content from single-nucleotide polymorphisms (SNPs) genotyped by microarray. Furthermore, in organ or stem cell transplantation setting the KIR locus offers additional genetic information for donor selection and prediction of clinical outcome (Cooley et al., 2010; Impola et al., 2014; Littera et al., 2017), and for many of these clinical genome datasets SNP microarray provides the most cost-effective genotyping platform as well. To date, several KIR copy number or gene content analysis methods have been implemented for sequencing data (Norman et al., 2016; Maniangou et al., 2017; Wagner et al., 2018; Chen et al., 2020; Roe \& Kuang, 2020), but to our knowledge only one SNP-based approach exists so far (Vukcevic et al., 2015). These approaches reach a high accuracy which makes KIR inference reliable enough for research and even practical clinical applications. However, regarding biobank data, a stand-alone application that does not require 
82

submitting individual genotype data to external servers would be essential. To this end, we have implemented a random forest (RF) based KIR gene content prediction in the R environment exploiting SNP data. The reference data used for model fitting comprises KIR genotypes determined by targeted sequencing and 5774 genotyped SNPs in the KIR chromosomal region. Based on prediction of an independent subset of data, our results demonstrate a mean overall accuracy of $99.2 \%$ which is comparable to previously published methods.

\section{Materials and Methods}

\section{Subjects}

Blood donor genomic DNA and genotype data were obtained from the Blood Service Biobank, Helsinki, Finland. The samples were collected from Finnish blood donors who had given a written informed consent for biobank research according to the Finnish Biobank Act (688/2012) supervised by the Finnish Medicines Agency (Fimea). The use of the biobank data in this study was approved by the Blood Service Biobank (002-2018).

\section{Genotyping}

Genotyping of samples was originally performed on a customized ThermoFisher Axiom array at the Thermo Fisher genotyping service facility (San Diego, USA) as a part of the FinnGen project. After the embargo period, imputed genotypes were returned to the Blood Service Biobank. Genotypes from altogether 807 samples with KIR type data were available for this study.

Genotype calling and quality control steps are described in

https://finngen.gitbook.io/documentation/methods/genotype-imputation. The array marker files can be downloaded from www.finngen.fi/en/researchers/genotyping. The protocol for genotype data liftover to hg38/GRCh38 is described in detail in www.protocols.io/view/genotyping-chip-data-lift-over-toreference-genome-xbhfij6?version warning=no, and genotype imputation protocol is described in www.protocols.io/view/genotype-imputation-workflow-v3-0-xbgfijw. The FinnGen array was designed to provide an imputation grid to cover as much of the genetic variation as possible. The SNP content in 
104 the KIR region (chr19 54.4-55.1 Mb, GRCh38/hg38) used in this study was generated by the FinnGen

105 imputation and quality control pipeline described above.

106 KIR genotyping at absence-presence level of 818 samples was purchased from Histogenetics LLC (NY, 107 USA) in two parts. The first randomly selected set of 473 biobank samples was complemented with 108 another set of 345 samples to ensure that each KIR gene and both haplotype groups occurred at high 109 enough frequencies in the reference data. Altogether $16 \mathrm{KIR}$ genes were genotyped: 2DL1, 2DL2, 2DL3, 110 2DL5, 2DP1, 2DS1, 2DS2, 2DS3, 2DS4, 2DS5, 3DL1, 3DS1, 2DL4, 3DL2, 3DL3, and 3DP1.

Imputation models

113

The SNP genotype and KIR typing data were combined into a single data set consisting of 807 KIR typed and SNP genotyped samples. SNP variants in the KIR region with missing data and KIR genes that showed zero variance were excluded from analysis. Based on the latter criterion, the KIR framework genes 2DL4, 3DL2, 3DL3, and 3DP1 were not included.

The outline of the modelling set up is depicted in Figure 1. The random forest model for predicting KIR gene content was implemented with R v4.0.4 (R Core Team, 2021) using the library ranger v0.12.1 (Wright \& Ziegler, 2017). The model error was estimated by dividing the original 818 samples randomly into two approximately equal subsets to one of which a RF model for each KIR gene was fitted while the other subset was used for prediction with the fitted model. SNP dosage values in the KIR region on chr19 were used as predictor variables, and the KIR gene content ( 1 for presence, 0 for absence) as determined by targeted sequencing served as the target phenotype variable. Feature selection within the model fitting was implemented using the permutated importance metric. Variants achieving an importance $>1 \times 10^{-5}$ were accepted into the model. The final model was fitted with the full dataset

127 (Figure 1).

Accuracy metrics were calculated using the R library caret v6.0-86 (Kuhn, Max, 2020). Positive predictive value (PPV) was defined as (sensitivity * prevalence) / ((sensitivity*prevalence) + ((1-specificity)*(1prevalence))). Negative predictive value (NPV) was defined as (specificity * (1-prevalence)) / ((1sensitivity $) *$ prevalence $)+(($ specificity $) *(1$-prevalence $)))$. Balanced accuracy was calculated as 
133 and $95 \%$ confidence intervals for the accuracy estimates were determined by binomial distribution. The

134 data were managed with the tidyverse v1.3.0 (Wickham et al., 2019) package system.

135 To compare our method with KIR*IMP, KIR*IMP v1.2.0 (http://imp.science.unimelb.edu.au/kir/)

136 (Vukcevic et al., 2015) was applied to the 818 samples constituting our reference panel. Prior to

137 submitting the data to KIR*IMP, the genotypes were transferred to hg19 coordinates with UCSC LiftOver

138 (http://genome.ucsc.edu/cgi-bin/hgLiftOver) and phased with shapeit v2.r904 (O'Connell et al., 2014)

139 with default parameters except for burn $=10$, prune $=10$, main $=50$ and window $=0.5$. The orientations of

140 the SNPs in the phased dataset were harmonised according to the KIR*IMP SNP information file

141 (http://imp.science.unimelb.edu.au/kir/static/kirimp.uk1.snp.info.csv) using a custom R script. In

142 analysing the results, we used KIR2DS4TOTAL and KIR3DL1ex9 CNV imputation results of KIR*IMP to

143 compare with our KIR2DS4 and KIR3DL1 absence-presence imputations, respectively.

Code and data availability

146

147

The analysis code and the models are available at https://github.com/FRCBS/KIR-imputation. SNP genotype data have been deposited at the European Genome-phenome Archive (EGA), which is hosted by the $\mathrm{EBI}$ and the CRG, under accession number EGAS00001005457. Further information about EGA can be found on https://ega-archive.org "The European Genome-phenome Archive of human data consented for biomedical research" (http://www.nature.com/ng/journal/v47/n7/full/ng.3312.html).

\section{Results}

154

Accuracy estimates for each KIR gene for prediction of an independent test set are listed in Table 1. In summary, the mean overall accuracy of prediction was 0.985 (95\% $\mathrm{Cl} 0.970-0.992)$. The lowest accuracy of 0.956 (95\% Cl 0.937-0.973) was obtained for KIR2DL5 while KIR2DL2, KIR2DL3, KIR2DS2, KIR2DS4 and KIR3DL1 all achieved an overall accuracy of 1 . Accuracy estimates for the test data and the RF out-of-bag (training set and full data) are plotted in Figure 2a. SNPs used by the models are listed in Supplementary Table 1.

161 To compare our approach with KIR*IMP, we converted our dataset of 807 samples to hg19 genome 162 build and harmonised the SNP orientations. 126 SNPs out of 5900 could not be lifted over to hg19, and 
163 out of the 301 SNPs used by KIR*IMP 249 were found in our input data. SNP allele frequencies between

164 the KIR*IMP reference panel and the input data had Pearson's correlation coefficient of 0.968

165 (Supplementary Figure 1a). Mean accuracy based on an estimate from the KIR*IMP reference subsetted 166 for the input SNPs was 96.59\% (Supplementary Figure 1b). Accuracy metrics for the imputation of our 167 data by KIR*IMP are indicated by grey colour in Figure 2a. In summary, for all 12 included KIR genes we 168 observed a distinctly lower imputation accuracy for KIR*IMP in comparison with our method.

169 To compare the level of overall accuracy of our SNP-based method with an established sequencing170 based approach, we extracted the results of the evaluation by Chen and co-workers (Chen et al., 2020)

171 for the KIR imputation method kpi (Roe \& Kuang, 2020). Figure 2b shows the accuracy of kpi compared 172 with our test set results. The observed values were highly similar with KIR2DS3 being the most difficult 173 gene to impute correctly.

174 Varying numbers of missing SNPs within the KIR region reduced the imputation accuracy in accordance 175 with the fraction of removed variants. At $80 \%$ of the SNPs present the accuracy generally remained at a 176 good level but started to increasingly deteriorate after that (Figure 2c).

177 Imputation posterior probability (PP) values are potentially informative of imputation uncertainty and 178 can be incorporated into association analyses (Zhou et al., 2020). Figure $2 d$ shows the PP distributions 179 for each imputed KIR gene. Figure 2e shows confusion tables for KIR gene absence/presence 180 classifications. Here, the presence of each imputed KIR gene was determined by the criterion PP>0.5; 181 otherwise the gene was determined to be absent.

182 Figure 3a shows the effect of PP-based filtering on imputation accuracy. More stringent PP thresholds 183 slightly improved accuracy estimates for KIR2DL1, KIR2DP1, KIR2DS3, KIR2DS5 and KIR3DS1. The number 184 of samples discarded as a result of PP filtering varied between genes (Figure 3b); for about half of the 185 KIR genes the filtering had no effect.

\section{Discussion}

Genome data generated in a growing number of biobank projects is instrumental to detailed immunogenetic analyses of several clinical phenotypes and diseases. Within the current technological and economical constraints SNP microarrays offer a practical way for genotyping hundreds of thousands of individuals. The KIR gene content, despite being a relatively coarse-scale feature, has been shown to 
193 influence many immune-mediated disorders (Bashirova et al., 2006; Parham \& Guethlein, 2018) and

194 complications in pregnancy (Colucci, 2017). Imputation of KIR gene content from SNPs in a scalable way

195 is therefore essential to analysing and interpreting large biomedical databases. To this end, in the

196 present study we have built a machine learning model for inferring KIR gene content from SNP dosage

197 data for stand-alone application in biobanks and other clinical data collections. Exploitation of random

198 forest for imputing KIRs from SNP genotypes was first implemented in the KIR*IMP software (Vukcevic

199 et al., 2015), which runs on a remote server (http://imp.science.unimelb.edu.au/kir/). The main

200 difference of our method in comparison with KIR*IMP is that it does not require phased data, the

201 models can be downloaded and run locally, and the user can build their own models. However, KIR*IMP

202 produces a more detailed output that includes A and B haplotypes, framework genes KIR3DP1 and

203 KIR2DL4, variants of KIR2DS4 and KIR3DL1 and gene copy numbers. Otherwise, at the level of gene

204 absence-presence, the imputation accuracy of our method compares favourably not only with KIR*IMP

205 but also to sequencing-based methods (Chen et al., 2020; Roe \& Kuang, 2020).

206 In all imputation evaluations KIR2DS3 demonstrated the largest error in overall accuracy, followed by

207 KIR2DL5 and KIR2DS5. A common feature shared by these three genes is that their location within the

208 KIR chromosomal region is not fixed but can vary between centromeric and telomeric positions (Hsu et

209 al., 2002; Pyo et al., 2010). Conceivably, this kind of positional variance may confound the identification

210 of predictive SNPs resulting in greater imputation uncertainty. Other challenging genes were KIR2DL1

211 and KIR2DP1 which both harbour a relatively rare gene absence with population frequency of about

$2121.6 \%$, and therefore had few cases in the training data. In this regard, the out-of-bag estimate for the

213 whole dataset might be the most reliable error estimate for these genes, suggesting a balanced accuracy

214 and positive predictive value of about 0.95. Despite some challenges, KIR gene content imputation

215 presents a valuable tool for initial screening and provides a rational basis for further analyses.

216 Allelic diversity and homologous gene sequences make KIR typing challenging by NGS or microarray

217 probes. Moreover, the genetic complexity of the KIR region is also evidenced by segment duplications

218 which can result in haplotypes carrying more than two copies of a KIR gene (Norman et al., 2009). This

219 can negatively affect SNP-based imputation accuracy especially in rarer haplotypes that may not be well

220 represented in the model training data. Nevertheless, the large number of variants within the genomic

221 region allows extraction of information based on linkage with gene content, even if the causative

222 variants cannot in all cases be directly measured. This is also a shortcoming because linkage patterns

223 vary between populations and consequently models trained on one population may not be fully

Peer] reviewing PDF | (2021:08:64927:2:0:NEW 29 Nov 2021) 
224 transferrable to another. While the informative SNPs used by our method are not specific to the Finnish

225 population as such, but present a set of common genetic variants with relatively similar allele

226 frequencies across European populations, it is not guaranteed that the prediction would achieve as good

227 an accuracy in populations other than Finns. Therefore, for translating the method to other populations,

228 the best option would be building population-specific models or using a large training data set that

229 captures most of the existing genetic diversity in KIRs.

230 In estimating the model error we used cross-validation in a randomly selected half of our sample set.

231 The full published model, however, was trained on all samples, and estimating the error of the full

232 model is based solely on out-of-bag (OOB) samples from within the model bootstraps. Thus, we are

233 assuming that the $\mathrm{OOB}$ error captures the true model error at a reasonable accuracy. This assumption

234 was supported by our data from the cross-validation where the OOB error from the training set closely

235 followed the estimate from the independent test set. Furthermore, as the full model is trained on all the

236 samples, it can be assumed to better capture the KIR diversity present in the population.

237 Our method is also limited by the requirement of the availability of informative SNPs in the dataset 238 under analysis. These variants are not genotyped by all microarrays commonly used in genome analysis

239 and therefore selection of a suitable platform is crucial. Another noteworthy limitation is that the 240 method is not capable of identifying alleles. To date, only targeted sequencing-based approaches can

241 resolve KIR alleles (Norman et al., 2016; Maniangou et al., 2017; Wagner et al., 2018; Roe \& Kuang, 242 2020).

243

244

\section{Conclusions}

245

246

Linkage disequilibrium patterns vary between human populations and potentially affect imputation of complex genotypes including KIR variation. Here we have studied KIR gene content imputation in the Finnish population to build a method suitable for biobank data and to evaluate the advantages of a population-specific reference. The results based on cross-validation demonstrate a good overall accuracy and highlight the importance of matching the imputation reference panel to the target population. As KIR function is modified by considerable allelelic diversity, a possible future direction is to extend KIR imputation from SNPs to cover allelic diversity for more detailed analysis of immunogenetic associations. 
We thank the Finnish Red Cross Blood Service Biobank and the blood donors for providing the samples.

258 FinnGen is acknowledged for providing the SNP genotype data.

259

260

261

262

263

264

265

\section{Competing interests}

The authors declare no competing interests.

\section{References}

Bashirova AA, Martin MP, McVicar DW, Carrington M. 2006. The Killer Immunoglobulin-Like Receptor Gene Cluster: Tuning the Genome for Defense. Annual Review of Genomics and Human Genetics 7:277-300. DOI: 10.1146/annurev.genom.7.080505.115726.

Bontadini A, Testi M, Cuccia M, Martinetti M, Carcassi C, Chiesa A, Cosentini E, Dametto E, Frison S, lannone A, Lombardo C, Malagoli A, Mariani M, Mariotti L, Mascaretti L, Mele L, Miotti V, Nesci S, Ozzella G, Piancatelli D, Romeo G, Tagliaferri C, Vatta S, Andreani M, Conte R. 2006. Distribution of killer cell immunoglobulin-like receptors genes in the Italian Caucasian population. Journal of Translational Medicine 4:44. DOI: 10.1186/1479-5876-4-44.

Carr WH, Pando MJ, Parham P. 2005. KIR3DL1 Polymorphisms That Affect NK Cell Inhibition by HLA-Bw4 Ligand. The Journal of Immunology 175:5222-5229. DOI: 10.4049/jimmunol.175.8.5222.

Chen J, Madireddi S, Nagarkar D, Migdal M, Vander Heiden J, Chang D, Mukhyala K, Selvaraj S, Kadel EE, Brauer MJ, Mariathasan S, Hunkapiller J, Jhunjhunwala S, Albert ML, Hammer C. 2020. In silico tools for accurate HLA and KIR inference from clinical sequencing data empower immunogenetics on individual-patient and population scales. Briefings in Bioinformatics:bbaa223. DOI: 10.1093/bib/bbaa223. 
Colucci F. 2017. The role of KIR and HLA interactions in pregnancy complications. Immunogenetics 69:557-565. DOI: 10.1007/s00251-017-1003-9.

Cooley S, Weisdorf DJ, Guethlein LA, Klein JP, Wang T, Le CT, Marsh SGE, Geraghty D, Spellman S, Haagenson MD, Ladner M, Trachtenberg E, Parham P, Miller JS. 2010. Donor selection for natural killer cell receptor genes leads to superior survival after unrelated transplantation for acute myelogenous leukemia. Blood 116:2411-2419. DOI: 10.1182/blood-2010-05-283051.

Frazier WR, Steiner N, Hou L, Dakshanamurthy S, Hurley CK. 2013. Allelic Variation in KIR2DL3 Generates a KIR2DL2-like Receptor with Increased Binding to its HLA-C Ligand. The Journal of Immunology 190:6198-6208. DOI: 10.4049/jimmunol.1300464.

Garcia-Beltran WF, Hölzemer A, Martrus G, Chung AW, Pacheco Y, Simoneau CR, Rucevic M, LamotheMolina PA, Pertel T, Kim T-E, Dugan H, Alter G, Dechanet-Merville J, Jost S, Carrington M, Altfeld M. 2016. Open conformers of HLA-F are high-affinity ligands of the activating NK-cell receptor KIR3DS1. Nature Immunology 17:1067-1074. DOI: 10.1038/ni.3513.

Hsu KC, Chida S, Geraghty DE, Dupont B. 2002. The killer cell immunoglobulin-like receptor (KIR) genomic region: gene-order, haplotypes and allelic polymorphism: Hsu et al · KIR genomic region and KIR haplotypes. Immunological Reviews 190:40-52. DOI: 10.1034/j.1600065X.2002.19004.x.

Impola U, Turpeinen H, Alakulppi N, Linjama T, Volin L, Niittyvuopio R, Partanen J, Koskela S. 2014. Donor Haplotype B of NK KIR Receptor Reduces the Relapse Risk in HLA-Identical Sibling Hematopoietic Stem Cell Transplantation of AML Patients. Frontiers in Immunology 5. DOI: 10.3389/fimmu.2014.00405.

Ivarsson MA, Michaëlsson J, Fauriat C. 2014. Activating Killer Cell Ig-Like Receptors in Health and Disease. Frontiers in Immunology 5. DOI: 10.3389/fimmu.2014.00184.

Kuhn, Max. 2020. caret: Classification and Regression Training.

Lanier LL. 2008. Up on the tightrope: natural killer cell activation and inhibition. Nature Immunology 9:495-502. DOI: 10.1038/ni1581. 
Littera R, Piredda G, Argiolas D, Lai S, Congeddu E, Ragatzu P, Melis M, Carta E, Michittu MB, Valentini D, Cappai L, Porcella R, Alba F, Serra M, Loi V, Maddi R, Orrù S, La Nasa G, Caocci G, Cusano R, Arras M, Frongia M, Pani A, Carcassi C. 2017. KIR and their HLA Class I ligands: Two more pieces towards completing the puzzle of chronic rejection and graft loss in kidney transplantation. PLOS ONE 12:e0180831. DOI: 10.1371/journal.pone.0180831.

Maniangou B, Legrand N, Alizadeh M, Guyet U, Willem C, David G, Charpentier E, Walencik A, Retière C, Gagne K. 2017. Killer Immunoglobulin-Like Receptor Allele Determination Using NextGeneration Sequencing Technology. Frontiers in Immunology 8:547. DOI: 10.3389/fimmu.2017.00547.

Maxwell LD, Wallace A, Middleton D, Curran MD. 2002. A common KIR2DS4 deletion variant in the human that predicts a soluble KIR molecule analogous to the KIR1D molecule observed in the rhesus monkey: Maxwell et al : A predicted soluble KIR2DS4 deletion variant. Tissue Antigens 60:254-258. DOI: 10.1034/j.1399-0039.2002.600307.x.

Middleton D, Gonzelez F. 2010. The extensive polymorphism of KIR genes. Immunology 129:8-19. DOI: 10.1111/j.1365-2567.2009.03208.x.

Norman PJ, Abi-Rached L, Gendzekhadze K, Hammond JA, Moesta AK, Sharma D, Graef T, McQueen KL, Guethlein LA, Carrington CVF, Chandanayingyong D, Chang Y-H, Crespi C, Saruhan-Direskeneli G, Hameed K, Kamkamidze G, Koram KA, Layrisse Z, Matamoros N, Mila J, Park MH, Pitchappan RM, Ramdath DD, Shiau M-Y, Stephens HAF, Struik S, Tyan D, Verity DH, Vaughan RW, Davis RW, Fraser PA, Riley EM, Ronaghi M, Parham P. 2009. Meiotic recombination generates rich diversity in NK cell receptor genes, alleles, and haplotypes. Genome Research 19:757-769. DOI: 10.1101/gr.085738.108.

Norman PJ, Hollenbach JA, Nemat-Gorgani N, Marin WM, Norberg SJ, Ashouri E, Jayaraman J, Wroblewski EE, Trowsdale J, Rajalingam R, Oksenberg JR, Chiaroni J, Guethlein LA, Traherne JA, Ronaghi M, Parham P. 2016. Defining KIR and HLA Class I Genotypes at Highest Resolution via High-Throughput Sequencing. The American Journal of Human Genetics 99:375-391. DOI: 10.1016/j.ajhg.2016.06.023. 
O’Connell J, Gurdasani D, Delaneau O, Pirastu N, Ulivi S, Cocca M, Traglia M, Huang J, Huffman JE, Rudan I, McQuillan R, Fraser RM, Campbell H, Polasek O, Asiki G, Ekoru K, Hayward C, Wright AF, Vitart V, Navarro P, Zagury J-F, Wilson JF, Toniolo D, Gasparini P, Soranzo N, Sandhu MS, Marchini J. 2014. A General Approach for Haplotype Phasing across the Full Spectrum of Relatedness. PLoS Genetics 10:e1004234. DOI: 10.1371/journal.pgen.1004234.

Parham P, Guethlein LA. 2018. Genetics of Natural Killer Cells in Human Health, Disease, and Survival. Annual Review of Immunology 36:519-548. DOI: 10.1146/annurev-immunol-042617-053149.

Pyo C-W, Guethlein LA, Vu Q, Wang R, Abi-Rached L, Norman PJ, Marsh SGE, Miller JS, Parham P, Geraghty DE. 2010. Different Patterns of Evolution in the Centromeric and Telomeric Regions of Group A and B Haplotypes of the Human Killer Cell Ig-Like Receptor Locus. PLoS ONE 5:e15115. DOI: 10.1371/journal.pone.0015115.

R Core Team. 2021. R: A language and environment for statistical computing. Vienna, Austria: $\mathrm{R}$ Foundation for Statistical Computing.

Roe D, Kuang R. 2020. Accurate and Efficient KIR Gene and Haplotype Inference From Genome Sequencing Reads With Novel K-mer Signatures. Frontiers in Immunology 11:583013. DOI: 10.3389/fimmu.2020.583013.

Stewart CA, Laugier-Anfossi F, Vely F, Saulquin X, Riedmuller J, Tisserant A, Gauthier L, Romagne F, Ferracci G, Arosa FA, Moretta A, Sun PD, Ugolini S, Vivier E. 2005. Recognition of peptide-MHC class I complexes by activating killer immunoglobulin-like receptors. Proceedings of the National Academy of Sciences 102:13224-13229. DOI: 10.1073/pnas.0503594102.

Vukcevic D, Traherne JA, Næss S, Ellinghaus E, Kamatani Y, Dilthey A, Lathrop M, Karlsen TH, Franke A, Moffatt M, Cookson W, Trowsdale J, McVean G, Sawcer S, Leslie S. 2015. Imputation of KIR Types from SNP Variation Data. The American Journal of Human Genetics 97:593-607. DOI: 10.1016/j.ajhg.2015.09.005.

Wagner I, Schefzyk D, Pruschke J, Schöfl G, Schöne B, Gruber N, Lang K, Hofmann J, Gnahm C, Heyn B, Marin WM, Dandekar R, Hollenbach JA, Schetelig J, Pingel J, Norman PJ, Sauter J, Schmidt AH, 
Lange V. 2018. Allele-Level KIR Genotyping of More Than a Million Samples: Workflow, Algorithm, and Observations. Frontiers in Immunology 9:2843. DOI: 10.3389/fimmu.2018.02843.

Wickham H, Averick M, Bryan J, Chang W, McGowan L, François R, Grolemund G, Hayes A, Henry L, Hester J, Kuhn M, Pedersen T, Miller E, Bache S, Müller K, Ooms J, Robinson D, Seidel D, Spinu V, Takahashi K, Vaughan D, Wilke C, Woo K, Yutani H. 2019. Welcome to the Tidyverse. Journal of Open Source Software 4:1686. DOI: 10.21105/joss.01686.

Wright MN, Ziegler A. 2017. ranger : A Fast Implementation of Random Forests for High Dimensional Data in C++ and R. Journal of Statistical Software 77. DOI: 10.18637/jss.v077.i01.

Wroblewski EE, Parham P, Guethlein LA. 2019. Two to Tango: Co-evolution of Hominid Natural Killer Cell Receptors and MHC. Frontiers in Immunology 10:177. DOI: 10.3389/fimmu.2019.00177.

Zhou W, Zhao Z, Nielsen JB, Fritsche LG, LeFaive J, Gagliano Taliun SA, Bi W, Gabrielsen ME, Daly MJ, Neale BM, Hveem K, Abecasis GR, Willer CJ, Lee S. 2020. Scalable generalized linear mixed model for region-based association tests in large biobanks and cohorts. Nature Genetics 52:634639. DOI: $10.1038 / \mathrm{s} 41588-020-0621-6$. 


\section{Figure 1}

Schematic presentation of the study setup.

The reference data set of 818 individuals was genotyped on the FinnGen SNP array, and KIR gene content was determined by targeted sequencing. We used random forests to fit models to the training data set comprising randomly selected 402 individuals. Feature selection was based on the importance metric computed through permutation. The model was re-fitted on SNPs achieving an importance of $>0$. Based on the test set comprising the other half of the samples $(n=405)$, we calculated prediction error estimates for the modeling approach. Finally, we used the whole data set $(n=807)$ to train complete models. 


\section{KIR reference data}

Targeted sequencing $(\mathrm{n}=818)$ :

- KIR gene absence/presence

Genotyping by FinnGen array ( $\mathrm{n=807):}$

- 5900 SNPs in the KIR region

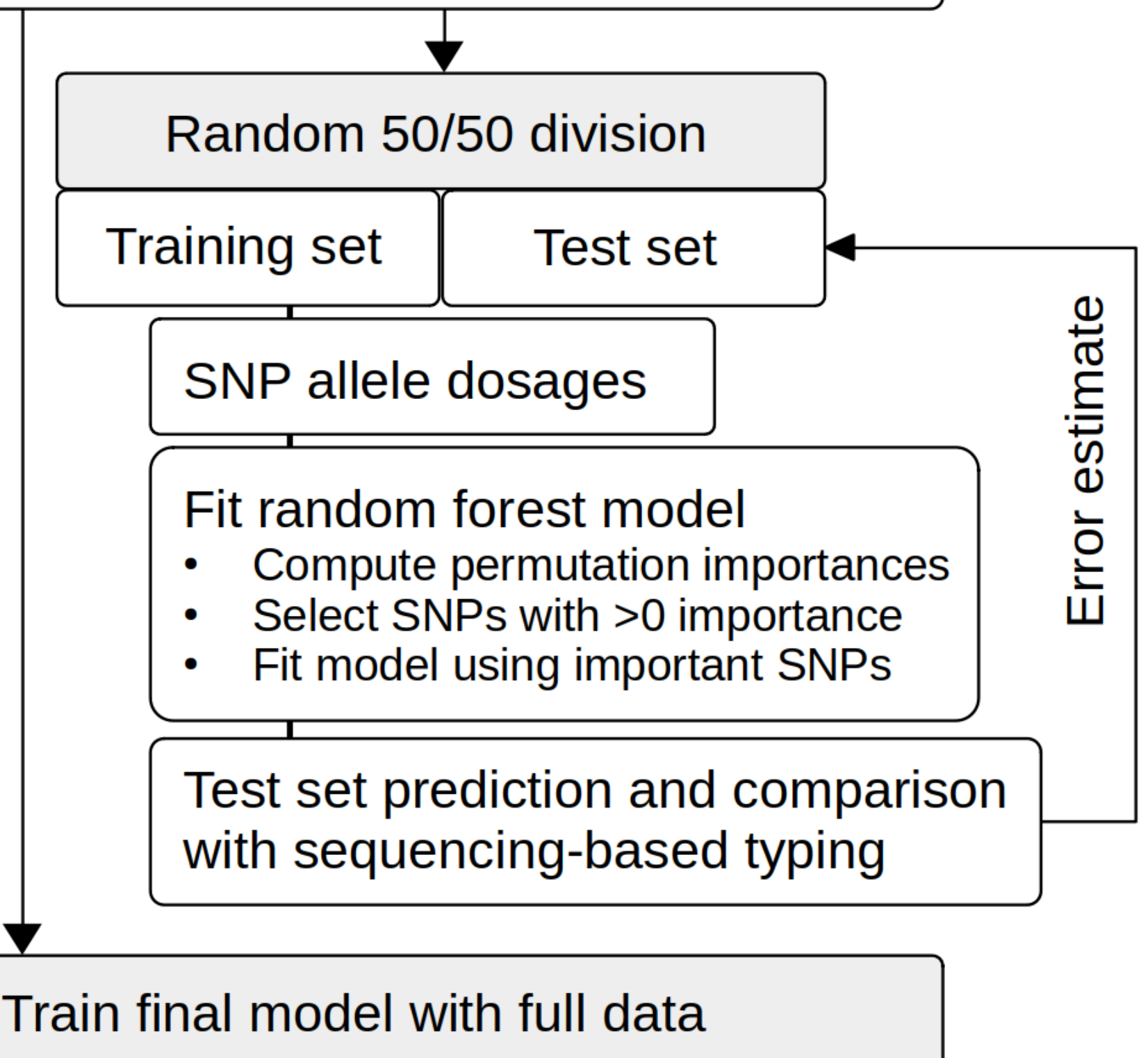




\section{Figure 2}

Overview of KIR prediction accuracy.

(A) Prediction performance metrics for the 12 imputed KIR genes. OOB: out-of-bag estimate from random forest models. Test set was predicted by models fitted on the training set. KIR*IMP was applied to the full dataset. Note the varying scale of the $y$-axis. (B) Comparison of overall accuracies between the test set and reported values for the WGS based method kpi extracted from the publication by Chen and co-workers. (C) Impact of missing SNPs on prediction performance in the test set. (D) Posterior probability distributions for test set prediction. Genotypes 0 and 1 denote absence and presence of a KIR gene, respectively. (E) Confusion tables for the test set prediction. Posterior probabilities $>0.5$ were classified as 'present'. 
A
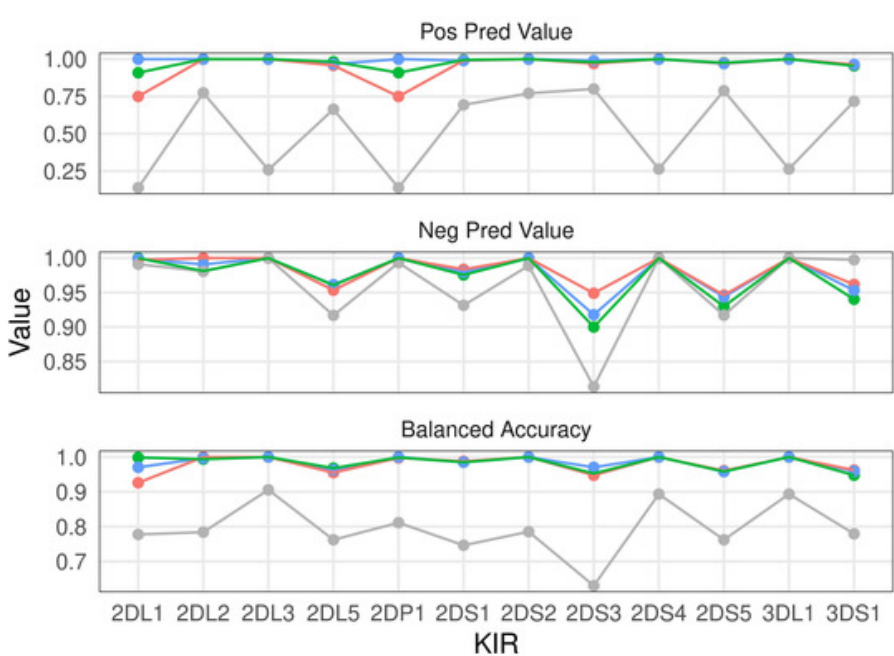

D
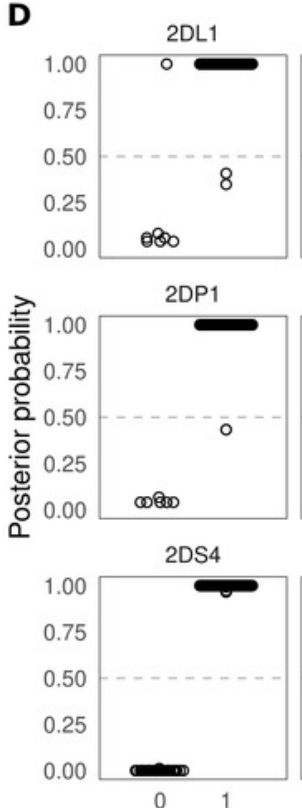

2DS1
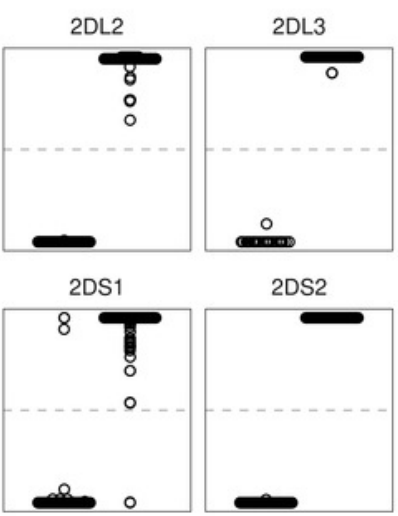

2DS2

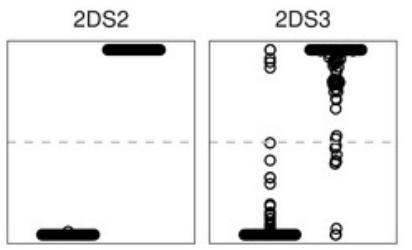

2DS5
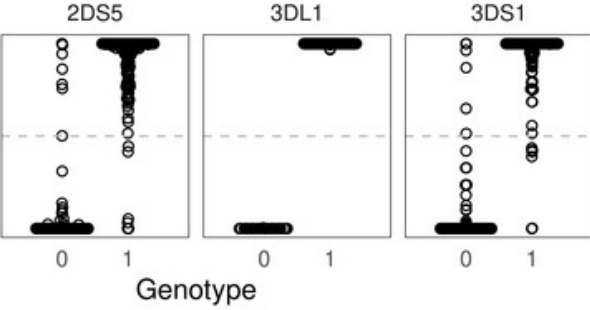

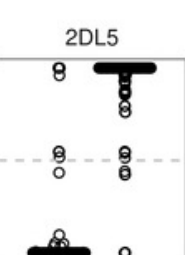

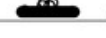

B

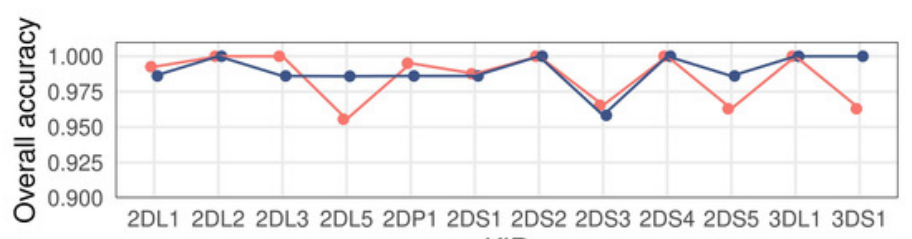

KIR

C

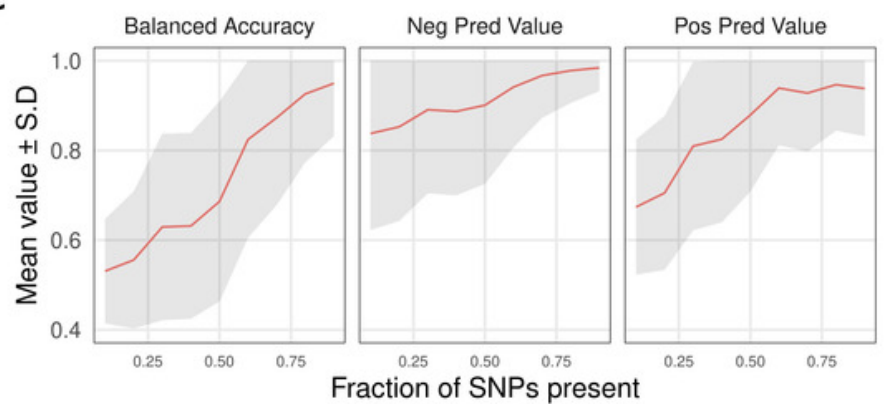

E
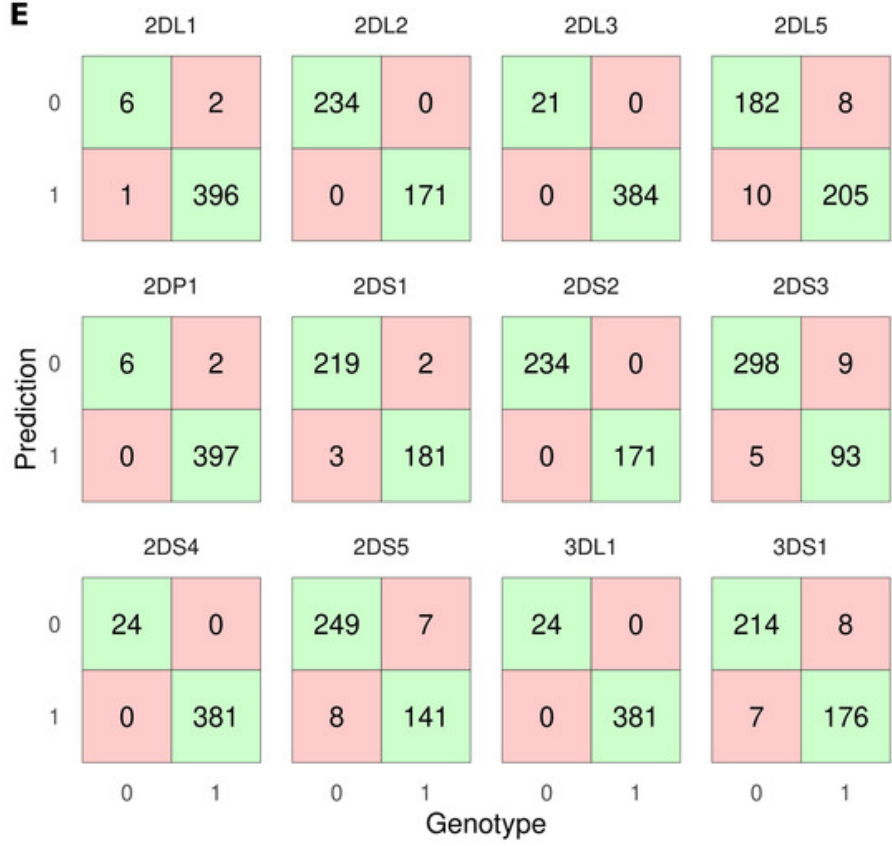

3DS1

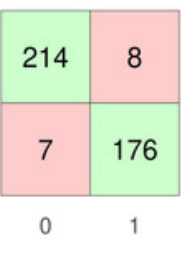


Figure 3

Effect of posterior probability on imputation accuracy.

Imputation results were filtered using varying posterior probability thresholds prior to calculating accuracies. The $x$-axis shows the threshold size relative to 0.5 : for example, a value of 0.1 sets the lower threshold for KIR gene presence at 0.6 and the upper threshold for gene absence at 0.4. (A) Accuracy estimates (y-axis) for the $12 \mathrm{KIR}$ genes at different threshold values. (B) Fraction of samples left (y-axis) after applying the thresholds.

A

— Balanced Accuracy — Neg Pred Value — Pos Pred Value
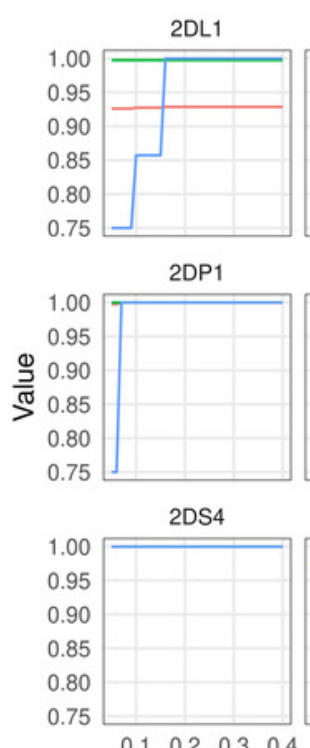

$\begin{array}{llllllllllllllll}0.1 & 0.2 & 0.3 & 0.4 & 0.1 & 0.2 & 0.3 & 0.4 & 0.1 & 0.2 & 0.3 & 0.4 & 0.1 & 0.2 & 0.3 & 0.4\end{array}$ PP threshold size
B

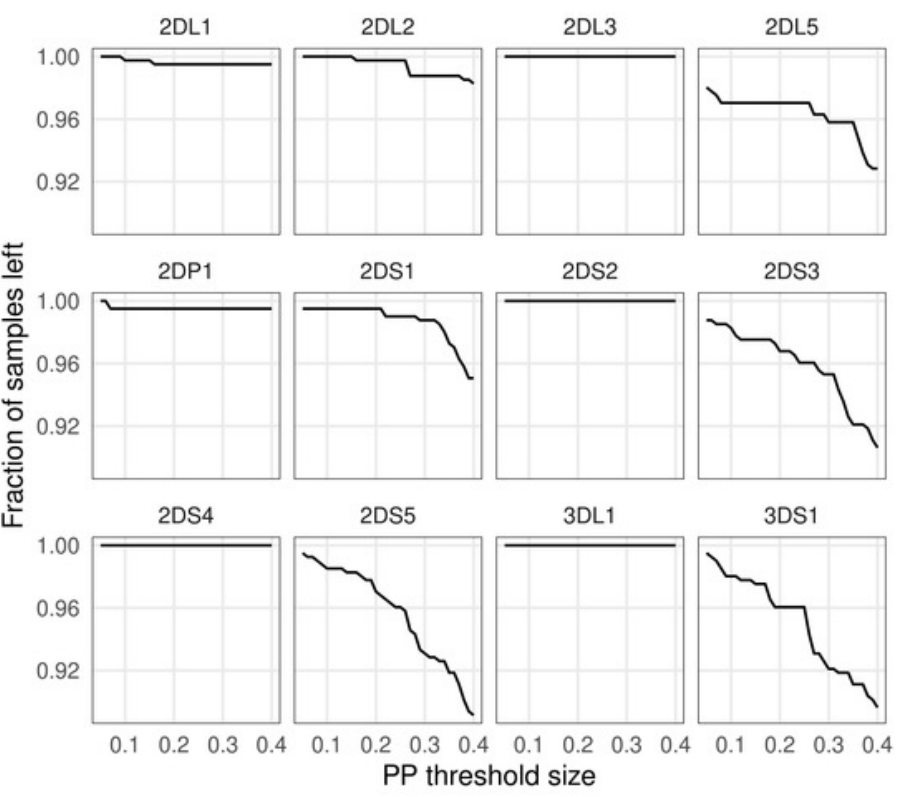


Table $\mathbf{1}$ (on next page)

KIR imputation accuracy. 


\begin{tabular}{|c|c|c|c|c|c|c|c|c|}
\hline KIR_gene & Sensitivity & Specificity & Pos.Pred.Value & Neg.Pred.Value & Precision & Recall & Balanced.Accuracy & Accuracy \\
\hline $2 \mathrm{DL} \overline{1}$ & 0.857 & 0.995 & 0.75 & 0.997 & 0.75 & 0.857 & 0.926 & 0.993 \\
\hline 2DL2 & 1 & 1 & 1 & 1 & 1 & 1 & 1 & 1 \\
\hline $2 \mathrm{DL3}$ & 1 & 1 & 1 & 1 & 1 & 1 & 1 & 1 \\
\hline 2DL5 & 0.948 & 0.962 & 0.958 & 0.953 & 0.958 & 0.948 & 0.955 & 0.956 \\
\hline 2DP1 & 1 & 0.995 & 0.75 & 1 & 0.75 & 1 & 0.997 & 0.995 \\
\hline 2DS1 & 0.986 & 0.989 & 0.991 & 0.984 & 0.991 & 0.986 & 0.988 & 0.988 \\
\hline 2DS2 & 1 & 1 & 1 & 1 & 1 & 1 & 1 & \\
\hline $2 \mathrm{DS} 3$ & 0.983 & 0.912 & 0.971 & 0.949 & 0.971 & 0.983 & 0.948 & 0.965 \\
\hline 2DS4 & 1 & 1 & 1 & 1 & 1 & 1 & 1 & 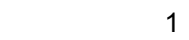 \\
\hline 2DS5 & 0.969 & 0.953 & 0.973 & 0.946 & 0.973 & 0.969 & 0.961 & 0.963 \\
\hline 3DL1 & 1 & 1 & 1 & 1 & 1 & 1 & 1 & \\
\hline 3DS1 & 0.968 & 0.957 & 0.964 & 0.962 & 0.964 & 0.968 & 0.962 & 0.963 \\
\hline
\end{tabular}

